\title{
Dolor neuropático: actualización en definiciones y su tratamiento farmacológico
}

\author{
Neuropathic pain: update in definitions and its pharmacological treatment
}

\author{
José Eduardo Bendaña
}

Residente de Primer Año, Postgrado de Anestesiología, Reanimación y Dolor (2020-2023), Facultad de Ciencias Médicas, UNAH, Tegucigalpa.

RESUMEN. El dolor neuropático definido como el dolor que se origina como consecuencia de una lesión en el sistema somatosensorial, incluye aproximadamente el $15 \%$ de las consultas por dolor. Debido a su diversa etiología y mecanismos fisiopatológicos, el dolor neuropático crea un reto para su manejo. Su relativa frecuencia en la atención primaria se convierte un tema que todo clínico deberá saber diagnosticar, tratar e identificar para su correcta remisión. Se realizó una revisión bibliográfica narrativa a partir de artículos originales, artículos de revisión y guías clínicas encontrados en bases de datos incluyendo PUBMED, HINARI, ScienceDirect y Cochrane Library, para el periodo 2010-2018. El diagnóstico del dolor neuropático se basa en la historia clínica. No se debe retrasar el alivio del dolor con medicamentos de primera línea como ser los antidepresivos tricíclicos e inhibidores de la recaptación de serotonina y noradrenalina.

Palabras clave: Dimensión del dolor, Dolor, Manejo del dolor, Neuralgia.
Recibido: 23-1-2019 Aceptado para publicación 14-10-2019

Dirección para correspondencia: Dr. JE Bendaña

Correo electrónico: jbendana92@gmail.com

CDeclaración de relaciones y actividades financieras y no financieras y conflictos de interés: ninguna.

DOI: https://doi.org/10.5377/rmh.v88i1.11591

\section{INTRODUCCIÓN}

El dolor funciona como sistema de alarma que permite protegernos de un daño actual, constituyendo así un mecanismo de defensa. El dolor neuropático definido por la Asociación Internacional para el Estudio del Dolor (IASP, International Association for the Study of Pain) es: "el dolor que se origina como consecuencia directa de una lesión o enfermedad que afecta el sistema somatosensorial". ${ }^{1}$ En revisiones sistemáticas se estima que la prevalencia a nivel global de dolor neuropático varia en un rango de $0.9 \%$ a $17.9 \% .^{2}$ En España se destacó que la prevalencia y visitas al médico por este tipo de dolor varía dependiendo de la causa del mismo; de igual manera se observaron similares proporciones entre hombres y mujeres exceptuando en polineuropatía en pacientes con diabetes tipo 2 donde se observó más frecuente en mujeres. ${ }^{3}$ En América Latina se estima que afecta al $2 \%$ de la población en donde 15 de cada 100 pacientes que acuden al médico por dolor es de tipo neuropatico. ${ }^{4}$ Entre las causas más comunes son: dolor lumbar con componente neuropático, neuropatía diabética, neuralgia post herpética y dolor neuropático como secuela postquirúrgica. ${ }^{1}$

Este tipo de dolor suele convertirse en dolor crónico el cual disminuye de manera significativa la calidad de vida del paciente y se ha asociado con alteraciones en la salud mental. ${ }^{1,2} \mathrm{Ha}$ existido gran interés en la comunidad científica tanto para el diagnóstico como el tratamiento del dolor neuropático ya que múltiples patologías traumáticas, metabólicas y crónico degenerativas son causa de este tipo de dolor. ${ }^{1,2}$

Esta revisión bibliográfica narrativa se ha realizado con el propósito de actualizar información sobre el dolor neuropático, su etiología, fisiopatología, diagnóstico y tratamientos farmacológicos actuales con el fin de ayudar al lector a diagnosticar y aliviar el dolor de origen neuropático. Se realizó búsqueda de artículos originales, artículos de revisión y guías clínicas en bases de datos incluyendo PUBMED, HINARI, ScienceDirect y Cochrane Library, para el periodo 2010-2018, con palabras claves como dolor, neuropático, neuralgia, manejo del dolor entre otras.

\section{DEFINICIÓN Y CLASIFICACIÓN}

El dolor neuropático es aquel que se origina como la consecuencia de lesión sobre las vías nerviosas somatosensoriales, este puede ser clasificado de varias maneras, las más 
comunes entre ellas de forma anatómica y de forma etiológica. La clasificación anatómica del dolor neuropático se clasifica como: central o periférico; siendo de origen central si la lesión es en el cerebro o médula espinal o de origen periférico si la lesión se encuentra en el nervio periférico, ganglio, plexo 0 raíz nerviosa ${ }^{3,4}$ (Ver cuadro 1). De estas es más común el dolor neuropático periférico que el central. Entre las causas más comunes de dolor neuropático periférico están: polineuropatía secundaria a diabetes y VIH, neuralgia postherpética, postquirúrgica y trauma de nervio periférico. ${ }^{2,5}$, Existe la necesidad de tener en cuenta el dolor neuropático de origen central ya que a pesar de no ser tan común como el de origen periférico, aun así, representa alrededor del $1-12 \%$ de los pacientes con antecedentes de ictus y lesiones a nivel cortical. ${ }^{5,6}$ Otros antecedentes importantes para la presencia de dolor neuropático central son aquellos con trauma raquimedular y esclerosis múltiple. ${ }^{7}$ Otra manera común de clasificar el dolor neuropático es de forma etiológica siendo esta: isquémica, degenerativa, traumática, infecciosa, inflamatoria, metabólica, neoplásica y tóxica. ${ }^{6,7}$

\section{FISIOPATOLOGÍA}

A diferencia del dolor nociceptivo, el cual ocurre cuando existe daño a un tejido activando nociceptores debido a mecanismos inflamatorios mediado por estímulos táctiles, térmicos y químicos y de esta manera transmitiendo impulsos nerviosos a las áreas superiores de procesamiento del dolor; el dolor neuropático agrupa una serie de condiciones que a pesar de sus diferencias de etiología y mecanismo de producción necesita cumplir el criterio de que exista lesión de la vía aferente somato sensorial para que este se desarrolle. ${ }^{8}$

Varios de estos mecanismos y no uno solo se pueden ver involucrados en la causa de dolor neuropático. Entre los mecanismos más estudiados para poder comprender la causa del dolor neuropático tenemos: la actividad neuronal ectópica, alteraciones en los canales de sodio y alteración en el neurotransmisor ácido gamma-aminobutírico (GABA). ${ }^{9,10}$

La presencia de dolor espontáneo y paroxístico en la ausencia de un estímulo externo es causado por actividad neuronal ectópica; esta ha sido observada mediante modelos animales y microneurografía en fibras aferentes en pacientes con neuropatía diabética. La actividad ectópica es clave para mantener los llamados síntomas positivos como ser el dolor espontáneo. ${ }^{9,10}$

Adicionalmente a la presencia de actividad ectópica posterior a una lesión nerviosa y que dicha actividad ectópica sea

Cuadro 1. Causas más comunes de dolor neuropático (Referencias 1,2,4).

\begin{tabular}{ll}
\hline Causas periféricas & Causas centrales \\
\hline Radiculopatía & Accidente cerebro vascular \\
Polineuropatía & Esclerosis Múltiple \\
Neuralgia Post-herpética & Lesión de Medula Espinal \\
Neuralgia Post-quirúrgica & \\
Trauma de Nervio periférico & \\
\hline
\end{tabular}

de manera persistente logra un cambio en la modulación de neurotransmisores nociceptivos secundarios y en donde la fosforilación del NMDA (ácido N-metil-D-aspartato) y AMPA (ácido a-amino-3-hidroxi-5-metilo-4-isoxazolpropiónico) y la sobreexpresión de canales de sodio logran disminuir el umbral necesario para la despolarización y propagación del impulso nervioso provocando dolor con estímulos que normalmente no serían dolorosos. ${ }^{10,11}$ Posterior a la lesión nerviosa y estimulo inflamatorio existe migración de macrófagos a nivel periférico y microglía a nivel central que genera liberación continua de citocinas proinflamatorias, incluyendo factor de necrosis tumoral, es así que se continúa con la estimulación de las terminaciones nerviosas y genera el dolor de tipo neuropatico. ${ }^{10}$

Con la presencia de la lesión nerviosa existe disminución de neuronas GABAérgicas, por lo que la inhibición en estos casos se vuelve deficiente ya sea mediante vías nerviosas inhibitorias descendentes e interneuronas en el asta posterior. ${ }^{10}$ De manera experimental las lesiones nerviosas disminuyen las concentraciones espinales de GABA y los receptores del mismo y en modelos animales se ha demostrado que con la administración exógena de GABA o benzodiacepinas disminuye la hipersensibilidad. ${ }^{11}$

\section{PRESENTACIÓN Y DIAGNÓSTICO}

El diagnóstico de dolor neuropático, a pesar de no contar con signos patognomónicos, se basa en la historia clínica y examen físico del paciente. Además, se pueden obtener datos complementarios que aporten al diagnóstico como cuestionarios, biopsia de nervios periféricos, estudios de imágenes y electrofisiología. ${ }^{11}$ Tanto el interrogatorio como examen físico deberá ir dirigido para poder diferenciar entre el dolor nociceptivo y el dolor neuropático para posteriormente descartar condiciones tratables, como por ejemplo secundario a compresión de nervio. ${ }^{8}$

Para poder diferenciar entre dolor nociceptivo y dolor neuropático es útil realizar un interrogatorio a profundidad sobre la calidad del dolor. El dolor neuropático suele contener ciertas características independientemente de su etiología. Los síntomas y signos de dolor neuropático pueden ser descritos como negativos o positivos y son el resultado del daño nervioso. Entre los síntomas positivos que más se encuentran en este tipo de pacientes tenemos: la hipersensibilidad, parestesias y sensación quemante 0 eléctrica. La hipersensibilidad podría ser de dos tipos ampliamente descritas; la alodinia que se describe como: "la sensación de dolor a estímulos inocuos" y la hiperalgesia que se describe como "la sensación aumentada de dolor frente a un estímulo doloroso". Entre los síntomas negativos podemos observar la ausencia de la sensibilidad en el área afectada y puede acompañarse con la presencia de afectación motora asociada a este tipo de dolor. $8,10,12$

El carácter del dolor es de suma importancia debido a que gran parte de los pacientes describen su dolor de manera inusual como quemante, fulgurante y demás disestesias. A pesar de que estas características no se presentan de manera universal, su presencia orienta a estudiar si se trata de dolor neuropático al igual que su causa. Durante el examen físico 
existe la necesidad de realizar pruebas de sensibilidad térmica, vibración, presión y medir el resultado del mismo.,10

Múltiples cuestionarios se han validado para determinar la presencia o ausencia de dolor de tipo neuropático entre ellos: el Leeds, el DN4, PainDETECT y el ID Pain, estos han alcanzado sensibilidad $(64 \%-85 \%)$ y especificidad $(74-90 \%)$ aceptables. Cabe mencionar que estos cuestionarios se basan en el reporte verbal de características de dolor como ser: disminución de la sensibilidad, presencia de sensación quemante y eléctrica más la opinión de un experto en dolor. ${ }^{13}$

Debido a la gran variedad de etiologías que causan dolor neuropático los exámenes complementarios deberán ir orientados al diagnóstico diferencial correspondiente a cada paciente. Entre los exámenes que se pueden realizar son: pruebas de química sanguínea como valorar los electrolitos y niveles de glucosa, imágenes biomédicas como tomografía y resonancia magnética. Existe la presencia de exámenes de biopsias de nervios y piel del área afectada en donde se ha encontrado mediante inmunohistoquímica alteraciones tanto neuronales como vasculares y uso de microneurografía para poder observar anormalidades en la conducción. Estos han servido para comprender la fisiopatología del mismo, sin embargo estos estudios tienen capacidad limitada para el uso clínico y han sido importantes para fines de investigación. Cabe recordar que el uso de exámenes complementarios no servirá para determinar la presencia o ausencia de dolor percibido por el paciente, sino que se utilizarán para tratar de definir la causa del mismo. 14-16

\section{TRATAMIENTO FARMACOLÓGICO}

El objetivo principal al momento de tratar el dolor neuropático es encontrar la causa del mismo y tratarla. El tratamiento oportuno ha mostrado disminuir la probabilidad de que el paciente presente dolor crónico en el futuro. ${ }^{16,17}$ Debido a la gran cantidad de fármacos utilizados para el tratamiento de dolor neuropático, en recientes años se han creado múltiples guías para ayudar al clínico escoger el fármaco indicado para cada paciente; entre las más utilizadas se encuentran: la guía IASP de la Asociación Internacional para el Estudio del Dolor del 2015 y la guía NICE del "National Institute of Health and Care Excellence" realizada en el 2017. ${ }^{18}$ Ambas guías establecen la Pregabalina, Gabapentina, los antidepresivos tricíclicos y los inhibidores de la receptación de serotonina y noradrenalina como fármacos de primera línea en el tratamiento de dolor neuropático. ${ }^{18,19}$ Existe el interés de utilizar fármacos combinados para tratar diferentes mecanismos del dolor neuropático, bajo esta premisa se han realizado estudios en donde han concluido, a pesar de utilizar grupos pequeños, que la combinación de fármacos opioides-anticonvulsivos y anticonvulsivos-antidepresivos fueron superiores a la monoterapia. ${ }^{19}$

\section{GRUPOS FARMACOLÓGICOS \\ Analgésicos}

Los analgésicos opioides y los antiinflamatorios no esteroideos a pesar de no ser de primera línea pueden ser útiles en los episodios agudos de dolor, en especial en la neuralgia postherpética. ${ }^{17}$ No se ha documentado alivio satisfactorio del dolor neuropático con el uso de antiinflamatorios no esteroideos por lo que se prefiere no utilizarlos de forma regular en este tipo de dolor. Se ha observado que los opioides necesitan dosis elevadas para producir analgesia aceptable y estos se evitan debido a que el uso prolongado del mismo, debido a la naturaleza del dolor, pueden producir dependencia, tolerancia y demás efectos adversos conocidos. Es por esto y por su falta de eficacia como monoterapia que los analgésicos opioides caen a la segunda línea de tratamiento del dolor neuropático. ${ }^{17-20}$

\section{Antidepresivos}

Los antidepresivos tricíclicos y los inhibidores de la receptación de serotonina y noradrenalina (IRSN) conforman la primera línea de tratamiento del dolor neuropático (Ver cuadro 2). Los antidepresivos tricíclicos actúan en los canales de sodio y son leves antagonistas de los receptores NMDA. La amitriptilina ha

Cuadro 2. Tratamiento farmacológico dolor neuropático (Referencias 18-20).

\begin{tabular}{|c|c|c|c|}
\hline Medicamento & Mecanismo de Acción & Dosis Diaria & Precaución \\
\hline \multicolumn{4}{|l|}{ Primera Línea } \\
\hline $\begin{array}{l}\text { Antidepresivos Triciclicos } \\
\text { Amitriptilina }\end{array}$ & $\begin{array}{l}\text { Inhibidores de recaptación de monoaminas, } \\
\text { leve inhibición canales de sodio }\end{array}$ & $25-150 \mathrm{mg}$ & $\begin{array}{l}\text { Pacientes cardiópatas, } \\
\text { Glaucoma, } \\
\text { Adenoma prostático }\end{array}$ \\
\hline \multicolumn{4}{|l|}{ IRSN } \\
\hline Duloxetina & $\begin{array}{l}\text { Inhibición recaptación de serotonina } \\
\text { y noradrenalina }\end{array}$ & $20-120 \mathrm{mg}$ & $\begin{array}{l}\text { Hepatopatía } \\
\text { Hipertensión }\end{array}$ \\
\hline Venlaflaxina & & $150-250 \mathrm{mg}$ & Cardiopatía \\
\hline \multicolumn{4}{|l|}{ Antiepilépticos } \\
\hline Gabapentina & $\begin{array}{l}\text { Inhibición subunidad Alfa2Delta canales } \\
\text { de calcio. }\end{array}$ & $900-3600 \mathrm{mg}$ & Insuficiencia Renal \\
\hline Pregabalina & & $150-600 \mathrm{mg}$ & \\
\hline \multicolumn{4}{|l|}{ Segunda Línea } \\
\hline Tramadol & Agonista receptor $\mathrm{Mu}$ & Max 400mg & $\begin{array}{l}\text { Historia de abuso de sustancias } \\
\text { Tratamiento Prolongado }\end{array}$ \\
\hline
\end{tabular}


demostrado en múltiples estudios su eficacia y seguridad para el tratamiento de dolor neuropático. ${ }^{17}$ Su acción es más rápida y su dosis diaria es menor que en su indicación para la depresión. Los efectos adversos más asociados a los antidepresivos tricíclicos son: sedación, efectos anticolinérgicos e hipotensión ortostática. ${ }^{18,19}$ Se recomienda la realización de un electrocardiograma previo al inicio de antidepresivos tricíclicos, en especial en adultos mayores. El mecanismo de acción de los inhibidores de la receptación de serotonina y noradrenalina aumenta el control inhibitorio y de esa manera reduce el dolor neuropático. El fármaco más estudiado de este grupo es la duloxetina que ha sido eficaz. ${ }^{17-19}$ Entre los efectos adversos más comunes de este grupo de fármacos son: náusea y dolor abdominal. Ambos tipos de antidepresivos reducen el dolor crónico y de manera adicional son beneficiosos cuando existe depresión como comorbilidad. ${ }^{8,17,18}$

\section{Antiepilépticos}

El uso de antiepilépticos también es considerado de primera línea en especial el uso de Gabapentina y Pregabalina para el uso de dolor neuropático periférico; al igual que el uso de Carbamazepina que es el fármaco de primera línea en la presencia de neuralgia del trigémino. ${ }^{17-20}$ Estudios demuestran que la Gabapentina y la Pregabalina son al menos igual de efectivos que la Amitriptilina. ${ }^{21}$ Los efectos adversos reportados por estos medicamentos generalmente son tolerables y entre ellos se encuentran: náusea, cefalea, mareos y ataxia. Se cree que su efectividad en el dolor neuropático se debe a disminución de la sensibilización a nivel central debido a su unión a la subunidad alfa 2 delta de los canales de calcio al igual que su influencia en la neurotransmisión de GABA. ${ }^{18-22}$

\section{CONCLUSIÓN}

El diagnóstico y tratamiento del dolor neuropático es complejo, sin embargo, se han realizado grandes avances y se está a la espera de nuevos tratamientos y métodos diagnósticos. El fin de cada médico frente a un paciente con dolor neuropático será siempre el alivio del mismo, diagnosticar su causa y su referencia a un nivel superior siempre que sea necesario. Resulta arduo individualizar el tratamiento solo por sintomatología; el cual actualmente se basa en su respuesta terapéutica. Conviene cambio de medicamento o combinación entre clases si el resultado no es satisfactorio antes de continuar o llegar a su dosis máxima y así mejorar la tolerancia y efectividad de los mismos.

\section{REFERENCIAS}

1. Maritza Velasco V. Dolor neuropático. Rev Méd Clín Las Condes. 2014;25(4):625-34.

2. Van Hecke O, Austin SK, Khan RA, Smith BH, Torrance N. Neuropathic pain in the general population: a systematic review of epidemiological studies. Pain. 2014;155(4):654-62.

3. Gálvez R, Rejas J, Pérez M, Gómez M. Prevalencia del dolor neuropático en España: implicaciones clínicas, laborales y asistenciales. Med Clin. 2005;125(6):221-229.

4. Fernández R, Ahumada M, Muñoz R, Urra X, Yañez MV, Velasco M, et al. Guía para definición y manejo del Dolor Neuropático Localizado (DNL): Consenso Chileno. Rev El Dolor. 2011;No.55:12-31.

5. Bouhassira D. Neuropathic pain: definition, assessment and epidemiology. Rev Neurol (Paris). 2019; 175(1-2):16-25.

6. Martín Estefanía C. Protocolo diagnóstico y terapéutico del dolor neuropático. Medicine - Programa de Formación Médica Continuada Acreditado. 2015;11(78):4724-7.

7. Correa-lllanes G. Dolor neuropático, clasificación y estrategias de manejo para médicos generales. Rev Méd Clín Las Condes. 2014;25(2):189-99.

8. Reddi D, Curran N. Chronic pain after surgery: pathophysiology, risk factors and prevention. Postgrad Med J. 2014;90(1062):222-7; quiz 226Gilron I,

9. Baron R, Jensen T. Neuropathic pain: principles of diagnosis and treatment. Mayo Clin Proc. 2015;90(4):532-45.

10. Baron R, Binder A, Wasner G. Neuropathic pain: diagnosis, pathophysiological mechanisms, and treatment. Lancet Neurol. 2010;9(8):807-19.

11. Taylor BK. Pathophysiologic mechanisms of neuropathic pain. Current Pain Headache Rep. . 2001;5(2):151-61.

12. Jones RC, Lawson E, Backonja M. Managing neuropathic pain. Med Clin North Am. 2016;100(1):151-67.

13. Attal $N$, Bouhassira $D$, Baron R. Diagnosis and assessment of neuropathic pain through questionnaires. Lancet Neurol. 2018;17(5):456-66.

14. Lewis RP, Kriukelyte I. Complex neuropathic pain states. Anaesthesia\& Intensive Care Medicine. 2016;17(11):571-4.

15. Garcia-Larrea L, Hagiwara K. Electrophysiology in diagnosis and management of neuropathic pain. Rev Neurol (Paris). 2019;175(1-2):26-37..

16. Jongen JL, Hans G, Benzon HT, Huygen F, Hartrick CT. Neuropathic pain and pharmacological treatment. Pain Pract. 2014;14(3):283-95.

17. Attal N. Pharmacological treatments of neuropathic pain: The latest recommendations. Rev Neurol (Paris). 2019;175(1-2):46-50.

18. Fitzmaurice BC, Rayen ATA. Treatments for neuropathic pain: up-to-date evidence and recommendations. BJA Education. 2018;18(9):277-83.

19. Chaparro LE, Wiffen PJ, Moore RA, Gilron I. Combination pharmacotherapy for the treatment of neuropathic pain in adults. Cochrane Database Syst Rev. 2012(7):CD008943.

20. Duehmke RM, Derry S, Wiffen PJ, Bell RF, Aldington D, Moore RA. Tramadol for neuropathic pain in adults. Cochrane Database Syst Rev. 2017;6:CD003726

21. Moore RA, Straube S, Wiffen PJ, Derry S, McQuay HJ. Pregabalin for acute and chronic pain in adults. Cochrane Database Syst Rev. 2010(3):CD007076

22. Robertson K, Marshman LA, Plummer D. Pregabalin and gabapentin for the treatment of sciatica. J Clin Neurosci. 2016;26:1-7.

ABSTRACT. Neuropathic pain defined as pain that is caused by injury to the somatosensory system, includes approximately $15 \%$ of pain visits. Due to its diverse etiology and pathophysiological mechanisms, neuropathic pain creates a challenge for its management. Its relative frequency in primary care becomes a topic that every clinician should know how to diagnose, treat and identify for correct remission. A narrative bibliographic review was made in which original articles, review articles and clinical guidelines were searched in databases including PUBMED, HINARI, ScienceDirect and Cochrane Library, for the period 2010-2018. Diagnosis of neuropathic pain is based on medical history. Pain relief should not be delayed with frontline medications such as tricyclic antidepressants and serotonin and norepinephrine reuptake inhibitors.

Keywords: Neuralgia, Pain, Pain management, Pain measurement. 